\title{
Reciprocal causation mixture model for robust mendelian randomization analysis using genome- scale summary data
}

\section{Zipeng Liu}

The University of Hong Kong

\section{Yiming Qin}

The University of Hong Kong

\section{Tian Wu}

The University of Hong Kong https://orcid.org/0000-0002-0304-3992

\section{Justin Tubbs}

The University of Hong Kong https://orcid.org/0000-0002-6671-6816

\section{Larry Baum}

The University of Hong Kong

\section{Timothy Mak}

The University of Hong Kong

\section{Miaoxin Li}

Sun Yat San University

\section{Yan Zhang}

The University of Hong Kong https://orcid.org/0000-0002-5302-3690

Pak Sham ( $\sim$ pcsham@hku.hk)

The University of Hong Kong https://orcid.org/0000-0002-2533-7270

\section{Genetics Article}

Keywords: Mendelian randomization, GWAS, mixture model Reciprocal Causation Inference

Posted Date: August 17th, 2021

DOI: https://doi.org/10.21203/rs.3.rs-719945/v1

License: (c) (i) This work is licensed under a Creative Commons Attribution 4.0 International License. Read Full License 
Reciprocal causation mixture model for robust mendelian randomization analysis using genome-scale summary data

Zipeng Liu ${ }^{1,2,4 \#, ~ Y i m i n g ~ Q i n ~}{ }^{1,2,4 \#}$, Tian Wu ${ }^{1}$, Justin D. Tubbs ${ }^{1}$, Larry Baum ${ }^{1,2,4}$, Timothy Shin Heng Mak ${ }^{4}$, Miaoxin $\mathrm{Li}^{1,5,6^{*}}$, Yan Dora Zhang ${ }^{3,4^{*}}$, Pak Chung Sham ${ }^{1,2,4^{*}}$

1. Department of Psychiatry, Li Ka Shing Faculty of Medicine, The University of Hong Kong, Hong Kong SAR, China.

2. State Key Laboratory of Brain and Cognitive Sciences, The University of Hong Kong, Hong Kong SAR, China.

3. Department of Statistics \& Actuarial Science, Faculty of Science, The University of Hong Kong Hong Kong SAR, China

4. Centre for PanorOmic Sciences, Li Ka Shing Faculty of Medicine, The University of Hong, Hong Kong SAR, China.

5. Zhongshan School of Medicine, Center for Precision Medicine, Sun Yat-sen University, Guangzhou, China.

6. Key Laboratory of Tropical Disease Control (SYSU), Ministry of Education, Guangzhou, China.

\# These authors contribute equally to this work.

* Corresponding address: Pak Chung Sham: pcsham@hku.hk; Yan Dora

Zhang: doraz@hku.hk; Miaoxin Li: limiaoxin@mail.sysu.edu.cn 


\begin{abstract}
Mendelian randomization (MR) using GWAS summary statistics has become a popular method to infer causal relationships across complex diseases. However, the widespread pleiotropy observed in GWAS has made the selection of valid instrumental variables (IVs) problematic, leading to possible violations of MR assumptions and thus potentially invalid inferences concerning causation. Furthermore, current MR methods can examine causation in only one direction, so that two separate analyses are required for bi-directional analysis. In this study, we propose a novel strategy, $\mathrm{MRCl}$ (Mixture model Reciprocal Causation Inference), to estimate reciprocal causation between two phenotypes simultaneously using the genome-scale summary statistics of the two phenotypes and reference linkage disequilibrium (LD) information. Simulation studies, including strong correlated pleiotropy, showed that $\mathrm{MRCl}$ obtained nearly unbiased estimates of causation in both directions, and correct Type I error rates under the null hypothesis. In applications to real GWAS data, $\mathrm{MRCl}$ detected significant bi-directional and uni-directional causal influences between common diseases and putative risk factors.
\end{abstract}




\section{Introduction}

The advent of genome-wide association studies (GWASs) has confirmed widespread genetic correlations among numerous complex diseases and traits ${ }^{1}$. Such correlations may represent pleiotropic genetic effects on multiple phenotypes, or causal relationships between phenotypes ${ }^{2}$. The analysis of GWAS data may help to identify causal relationships between phenotypes, contributing to our understanding of disease aetiology. In recent years, causal modelling using Mendelian randomization (MR) has been widely applied to GWAS summary data ${ }^{3,4}$. MR uses genetic variants, typically single nucleotide polymorphisms (SNPs), as instrumental variables (IVs) which, to be valid, should be (1) associated with the exposure (the relevance assumption), (2) not associated with confounders of exposure and outcome (the independence assumption), and (3) only associated with outcome through exposure (the exclusion restriction assumption) ${ }^{5}$. Basically, MR estimates the causal effect of an exposure on an outcome by the ratio of the effect of a genetic variant on the outcome to the effect of the same genetic variant on the exposure. In practice, due to the high polygenicity of complex traits, multiple SNPs may be necessary to increase the power and robustness of $\mathrm{MR}^{6}$.

Many methods have been developed for causal estimation from multiple SNPs, including inverse-variance weights (IVW) ${ }^{4}$, Weighted Median ${ }^{7}$ and Weighted Mode ${ }^{8}$. However, using multiple SNPs as IVs also increases the chance of horizontal pleiotropy for some SNPs ${ }^{9}$, which would violate the exclusion restriction assumption. MR-Egger ${ }^{10}$ and MR-PRESSO ${ }^{11}$ address this issue under the InSIDE (instrument strength independent of direct effect) assumption, while more recent methods such as MRMix ${ }^{12}$ assume a normal-mixture model to consider horizontal pleiotropic effects. While these methods address the violation of the MR assumptions by either removing pleiotropic SNPs or explicitly modelling pleiotropic effects, they still involve the selection of independent IVs, which may exclude the majority of SNPs, with consequent loss of information. One recent advance is to account for correlated pleiotropy by estimating nuisance parameters of a mixture model from randomly selected genome-wide summary data ${ }^{13}$. This method uses linkage disequilibrium (LD) pruned variants for causation estimation. As with other existing methods, inference of the causal relationship in the reverse direction requires a separate analysis with a different set of IVs ${ }^{9,14}$.

In recent years, causal modelling based on MR approaches has been applied to investigate the inter-relationships between a wide range of phenotypes for which summary GWAS data are available ${ }^{15}$. With such widespread usage, and the proneness of MR methodologies to violations of model assumptions, there is a risk of false positive findings from such studies. False positive causal inferences may be particularly likely when most causal SNPs are pleiotropic so that few SNPs can serve as valid IVs ${ }^{16}$.

In this article, we propose a novel method, called $\mathrm{MRCl}$ (Mixture model Reciprocal Causal Inference) to infer the causal paths simultaneously in both directions between two phenotypes. Our method uses GWAS summary statistics of all available SNPs for the two phenotypes, together with reference LD information on the SNPs. 
We consider SNPs to fall into four mutually exclusive effects (trait-specific, pleiotropic and null SNPs) and construct a composite likelihood function that takes account of the LD among SNPs. Compared with existing MR approaches, our method tests for reciprocal causation without the selection of IVs, thus making full use of genetic information while explicitly modelling pleiotropy. In particular, $\mathrm{MRCl}$ is robust even in situations where most causal variants are pleiotropic. We applied $\mathrm{MRCl}$ to cardiovascular and metabolic disorders and some of their putative risk factors using public GWAS summary data, with results that provided novel insights into the causal relation between several pairs of phenotypes.

\section{Results}

\section{Overview of $\mathrm{MRCl}$}

All phenotypes and additively coded genotypes were assumed to have been standardized to have unit variance. In the full model, SNPs were assumed to fall into four mutually exclusive components: trait-specific $\left(G_{1}, G_{2}\right)$, pleiotropic $\left(G_{C}\right)$ and null SNPs $\left(G_{0}\right)$, with mixing proportions $\pi_{1}, \pi_{2}, \pi_{c}$ and $\pi_{0}$, respectively. SNPs in the $G_{1}$ and $G_{2}$ components have direct effect sizes of $\gamma_{1}$ and $\gamma_{2}$ on phenotype $Y_{1}$ and $Y_{2}$ respectively, while $G_{C}$ SNPs have direct effect sizes of $\gamma_{C 1}$ and $\gamma_{C 2}$ with covariance $\rho_{C 1, C 2}$, on $Y_{1}$ and $Y_{2}$. The effect sizes $\gamma_{1}, \gamma_{2}, \gamma_{C 1}$ and $\gamma_{C 2}$ were assumed to be normally distributed, and their variances were denoted as $\sigma_{1}^{2}, \sigma_{2}^{2}, \sigma_{C 1}^{2}$ and $\sigma_{C 2}^{2}$, respectively. Two reciprocal causal paths $\left(\delta_{12}\right.$ and $\left.\delta_{21}\right)$ were specified between $Y_{1}$ and $Y_{2}$ (Figure 1). Additionally, we considered systemic biases such as population stratification $\left(a_{1}\right.$ and $\left.a_{2}\right)$ and sample overlap $\left(\rho_{0}\right)$ in variance and covariance estimates for phenotype $Y_{1}$ and $Y_{2}$ in the model. From the above parameters, we also calculated the genetic correlation $\left(r_{g}\right)$ between the two phenotypes.

From these assumptions, we derived the bivariate distribution of the marginal effect size estimates of the two phenotypes from their respective GWAS. The covariance matrix of this bivariate distribution is determined by the mixture distribution of the direct effect sizes $\gamma$, the reciprocal causal effects $\delta$ and the nuisance parameters $a_{1}$, $a_{2}$ and $\rho_{0}$ (see Methods). We then calculated the composite likelihood function for all available SNPs to estimate the two causal effects as well as other nuisance parameters by an Expectation-Maximization (EM) algorithm, together with robust sandwich estimates of their standard errors. Besides the above full model scenario, we further considered four sub-model scenarios in which one or two components were absent (Supplementary Figure S1). Under these sub-model scenarios, we found that full model estimation often produced poor estimates, and developed a method based on model averaging to achieve more robust model fitting.

We then performed comprehensive simulations, including full model and sub-model scenarios, to evaluate the estimation accuracy, Type I error rate and statistical power of $\mathrm{MRCl}$, compared to existing MR methodologies.

\section{Simulation results}

\section{Estimation and hypothesis testing under the full model}

We compared the accuracy of the causal estimates between $\mathrm{MRCl}$ and IV-based MR methods under several simulated low polygenicity scenarios in which the true IVs 
could be considered as nearly independent to satisfy the assumption of classic MR methods. $\mathrm{MRCl}$ produced nearly unbiased estimates for both causal directions under both independent and correlated pleiotropy scenarios. Not surprisingly, using exposure-specific true causal SNPs as IVs in IV-based MR methods always obtained unbiased estimates. However, IV-based MR methods generated biased estimates when GWAS significant SNPs for the exposure phenotype were used as IVs, especially under scenarios with correlated pleiotropy. In these scenarios, excluding IVs which showed strong GWAS association with the outcome phenotype reduced the magnitude of the bias (Figure 2a-c and Supplementary Figure S2).

$\mathrm{MRCl}$ achieved correct control of the Type I error rates under all scenarios when the exposure phenotype had no effect on the outcome phenotype. Not surprisingly, IVbased MR methods also achieved correctly controlled of Type I error rates in the perfect scenario where all IVs were exposure-specific true causal SNPs. However, when IVs were selected according to statistical significance in GWAS of the exposure phenotype, most of the MR methods showed severely inflated Type I error rates in simulations with correlated pleiotropy. Excluding SNPs which showed strong association with the outcome phenotype decreased the Type I error rates, sometimes to the correct level (MR-Egger and Weighted Median), when correlated pleiotropy was present (Figure $2 \mathrm{~d}$ and Supplementary Table S2-S4).

In terms of statistical power under the alternative hypothesis, we only considered MR methods (MR-Egger, Weighted Mode and MRMix) that demonstrated correct control of Type I error rates when using statistically significant exposure-associated SNPs as IVs. MRCI and MR methods achieved comparable power in low polygenicity simulations: as high as almost 1 in the stronger causal direction and slightly lower in the weaker causal direction. For IV-based MR methods, excluding potential outcome-associated significant SNPs did not guarantee a sufficient power increase in correlated pleiotropy scenarios (Supplementary Table S3-S7).

Additionally, simulations of other scenarios, including high polygenicity and asymmetry between the two phenotypes (different levels of heritability and polygenicity, and where one phenotype was binary and the other quantitative) showed that $\mathrm{MRCl}$ achieved nearly unbiased estimates, well-controlled Type I error rates and favourable statistical power in both causal directions (Supplementary Table S8). Genetic correlation estimates calculated from the $\mathrm{MRCl}$ parameter estimates were highly consistent with those obtained from LD Score regression (LDSC) ${ }^{1}$ (Supplementary Table S9). Simulations with different sample sizes show that when the sample sizes of the GWAS of the two phenotypes are unequal, the accuracy of a causal effect estimate from the phenotype with the larger sample to the phenotype with the smaller sample is disproportionately reduced compared to the causal effect estimate in the other direction (Supplementary Table S10).

\section{Estimation and hypothesis testing under sub-models}

When the true model does not contain all four SNP components, there is a risk of incorrect inference from estimation that assumes the full model. To examine the robustness of $\mathrm{MRCl}$, we performed estimation under four sub-models which allowed 
for the absence of one or two specific components. Writing the full model as $S_{1,2, C}$ (indicating the presence of $Y_{1}$-specific SNPs, $Y_{2}$-specific SNPs, and pleiotropic SNPs, as well as the omnipresent null SNPs), the four sub-models were $s_{1, C}, s_{2, C}, s_{1,2}$ and $s_{C}$ (indicating the presence of the corresponding SNP components). We performed simulations to investigate model fitting performance under these sub-models, excluding $s_{1, C}$ as it is equivalent to $S_{2, C}$ (Supplementary Figure S1 and Table S1). We found that estimation assuming the full model often did not correctly infer the absent component, which resulted in biased causal effect estimates and inflated Type I error rates (Supplementary Figure S3a).

While estimation under the true model performed well in simulations, the true model is not known for applications to real data. Accordingly, to improve the robustness of $\mathrm{MRCl}$ we implemented an additional model fitting procedure which involved estimation under each sub-model and the full model, and performed model averaging of parameter estimates with weights that optimized the composite likelihood. Simulation results showed that this procedure usually assigned low weights to incorrect sub-models (Supplementary Figure S3b). When the model averaging composite likelihood was higher than the full model composite likelihood, which was usually the case when a sub-model is true (Supplementary Figure S3c), the parameter estimates from the model averaging procedure were adopted, instead of the full model estimates. In simulations under bi-directional, unidirectional and null causation models, this method gave nearly unbiased estimates of the reciprocal causal effects (Figure 3a), correct control of Type I error rates when a causal effect was absent, and acceptable power when a causal effect was present and the model included exposure-specific SNPs (Figure 3b).

\section{Comparison with CAUSE and MRMix}

CAUSE ${ }^{13}$ and MRMix ${ }^{12}$ are two recent methods which share similarities with $\mathrm{MRCl}$. With large samples (50,000 for both phenotypes), when the true model was $s_{1,2, C}$ or $s_{1,2}$, simulation results showed that these two methods produced unbiased estimates and correct Type I error rates. However, under scenarios where traitspecific SNPs were absent for one or both phenotypes (i.e., $s_{2, C}$ and $s_{C}$ ) these methods obtained biased causal estimates and inflated Type I error rates for the causal direction in which the exposure-specific SNPs were absent (Figure 4a-b). With small samples (20,000 for both phenotypes), CAUSE produced biased estimates and increased Type I error rates, even under the full model. Under these scenarios, both $\mathrm{MRCl}$ and MRMix maintained unbiased estimates and correct Type I error rates, with $\mathrm{MRCl}$ achieving slightly smaller estimation variance and greater statistical power (Figure 4c).

\section{Causation of risk factors on common diseases}

We applied $\mathrm{MRCl}$ to study causal relationships between 3 common diseases (coronary artery disease, CAD; ischemic stroke, IS; type 2 diabetes, T2D) and 16 putative risk factor phenotypes (Table 1 ). Figure 5 shows the estimated reciprocal causal effects $(\delta)$ between these diseases and phenotypes. Considering that 96 tests were performed, the Bonferroni adjusted significance level is $5.0 \times 10^{-4}$. The causal influences significant at this level were from low-density lipoprotein level (LDL) to 
$\operatorname{CAD}\left(\delta=0.33,95 \% \mathrm{Cl}=[0.17,0.47], \mathrm{p}\right.$-value $\left.=1.7 \times 10^{-5}\right)$, from diastolic blood pressure $(\mathrm{dBP})$ to IS $\left(\delta=0.19,95 \% \mathrm{Cl}=[0.12,0.26], \mathrm{p}\right.$-value $\left.=1.1 \times 10^{-7}\right)$, and from body mass index $(\mathrm{BMI})$ to T2D $\left(\delta=0.72,95 \% \mathrm{Cl}=[0.59,0.84]\right.$, $\mathrm{p}$-value $\left.=3.8 \times 10^{-30}\right)$. In the reverse direction, significant causal influences were detected from T2D to BMI ( $\delta=-0.17,95 \%$ $\mathrm{Cl}=[-0.24,-0.10], \mathrm{p}$-value $\left.=2.2 \times 10^{-6}\right)$, fasting glucose $(\delta=0.32,95 \% \mathrm{Cl}=[0.21,0.43]$, $\mathrm{p}$-value $\left.=4.8 \times 10^{-9}\right)$, and fasting insulin $(\delta=-0.23,95 \% \mathrm{Cl}=[-0.29,-0.17], \mathrm{p}$ value $=1.8 \times 10^{-15}$ ).

A number of nominally significant ( $p$-value $<0.05)$ causal effects that did not survive multiple testing adjustment were detected (Figure 5). These included effects of fasting glucose on CAD, LDL and pulse pressure on both IS and T2D. Nominally significant effects in the reverse direction included CAD on BMI, fasting insulin, LDL and $\mathrm{dBP}$, IS on triglycerides, and T2D on birth weight.

\section{Discussion}

We have developed a new method, $\mathrm{MRCl}$, to jointly estimate the reciprocal causal effects between two phenotypes using GWAS summary statistics and reference LD data. Our simulation studies, under both independent pleiotropy and correlated pleiotropy, indicate that $\mathrm{MRCl}$ obtains nearly unbiased estimates of causation in both directions, and maintains well-controlled Type I error rates under the null hypothesis, even when most causal SNPs for one or both phenotypes are pleiotropic. $\mathrm{MRCl}$ also achieves comparable statistical power to other methods when Type I error rates are controlled.

Our simulations showed that existing MR methods that require IVs selection produced unbiased estimates and correct Type I error rates when using genomewide significant exposure-associated IVs, for scenarios with independent pleiotropy. However, these methods often produced biased estimates and severely inflated Type I error rates in the presence of correlated pleiotropy. For MR-Egger ${ }^{10}$ and MRPRESSO ${ }^{11}$, designed to cope with horizontal pleiotropy under the InSIDE assumption, excluding potential outcome-associated SNPs according to a p-value cut-off helped control Type 1 error, but in practice the optimal choice for cut-off is unknown and may vary depending on the genetic architecture of the phenotypes as well as GWAS sample sizes. These are important limitations as pleiotropy is common for complex traits $^{11}$, and independent pleiotropy is implausible ${ }^{10}$.

The robustness of $\mathrm{MRCl}$ is partly derived from the explicit consideration of submodels with absent SNP components during model fitting. When a SNP component is absent, estimation under the full model sometimes wrongly infers the absent SNP components which can lead to spurious inferences on the causal effects. Thus, we perform estimation under both full model and sub-models, but the goal is not to select one best model. Rather, our aim is to obtain robust causal estimates. Based on simulation performance, our method involving model averaging reduces the chance of such spurious inferences and produces unbiased estimates and correct control of Type I error rates even in these challenging scenarios. Two recent methods, CAUSE ${ }^{13}$ and MRMix ${ }^{12}$, which share some similarities with $\mathrm{MRCl}$, nevertheless produced 
biased estimates and inflated Type 1 error rates when a trait-specific SNP component is absent.

$\mathrm{MRCl}$ also provides estimates for nuisance parameters such as the heritabilities and the genetic correlation, which may help clarify the genetic architecture of the two phenotypes. The genetic correlation estimates from $\mathrm{MRCl}$ are consistent with results obtained from LDSC. Moreover, our model takes the sample overlap into consideration, including complete overlap, which enables GWAS results obtained from the same samples (e.g., UK Biobank) to be analyzed for causal relationships.

When applied to real GWAS data, $\mathrm{MRCl}$ was able to identify several well-established causal relationships at a Bonferroni-corrected significance level, including a reciprocal causal relationship between body mass index and type 2 diabetes. Being overweight is known to be strongly associated with an increased risk of type 2 diabetes ${ }^{17-19}$, while weight loss is a known clinical feature of type 2 diabetes $^{20}$. $\mathrm{MRCl}$ also confirmed low-density lipoprotein level but not high-density lipoprotein level as a causal factor for coronary artery disease ${ }^{21-23}$, and diastolic blood pressure as a causal factor for ischemic stroke ${ }^{24}$. $\mathrm{MRCl}$ did not detect additional causal effects at the Bonferroni-corrected significance level may reflect its ability to control the Type I error rate. It is also possible that GWAS for some of the phenotypes may not have adequate statistical power because of the small sample size or high polygenicity.

The current formulation of $\mathrm{MRCl}$ has certain limitations. Our assumption of bivariate normality of standardized marginal effects in the four SNP components may not hold in practice, and serious violation of this assumption may affect the performance of the method. Further simulations to evaluate the sensitivity of the method to violations of this assumption is desirable. A second limitation is that we used composite likelihood for model fitting because marginal SNP effects are correlated. This necessitated the use of robust sandwich standard errors, which may lead to conservative, less powerful tests. Finally, in the scenario where pleiotropic effects are very strong and nearly no causal SNPs contribute specifically to the exposure, $\mathrm{MRCl}$ will typically produce very large standard errors for the causal path estimate from exposure to outcome, so that there is little power to detect such a causal effect even when it is present. However, this situation of having almost no SNPs that can be used as valid IVs could be a limitation for the MR approach in general, rather than for $\mathrm{MRCl}$ specifically.

\section{URLS}

PLINK software, https://www.cog-genomics.org/plink/1.9/;

GCTA software, http://cnsgenomics.com/software/gcta/;

TwoSampleMR software, https://github.com/MRCIEU/TwoSampleMR;

MR-PRESSO software, https://github.com/rondolab/MR-PRESSO;

MRMix software, https://github.com/gqi/MRMix;

LDSC software: https://github.com/bulik/ldsc;

CAUSE software: https://github.com/jean997/cause; 


\section{Methods}

\section{Data and full model}

$\mathrm{MRCl}$ uses the GWAS summary statistics of the studied two phenotypes ( $Y_{1}$ and $Y_{2}$ ) and reference LD information ( 1 million SNPs from 1000 Genome data). For a pair of reciprocal causal phenotypes (Figure 1a), each available SNP belongs to one of four mutually exclusive SNP components:

- $Y_{1}$-specific component $\left(G_{1}\right)$ : SNPs that contribute directly to $Y_{1}$ only;

- $Y_{2}$-specific component $\left(G_{2}\right)$ : SNPs that contribute directly to $Y_{2}$ only;

- pleiotropic component $\left(G_{C}\right)$ : SNPs that contribute directly to both phenotypes;

- null component $\left(G_{0}\right)$ : SNPs with no direct effects on either phenotype.

The proportions of all SNPs in the four components are $\pi_{1}, \pi_{2}, \pi_{c}$ and $\pi_{0}$. The values of the two phenotypes in an individual are given by:

$$
\begin{aligned}
& Y_{1}=\delta_{12} Y_{2}+\sum_{i \in G_{1}} \gamma_{1 i} X_{i}+\sum_{l \in G_{C}} \gamma_{C 1 l} X_{l}+e_{1} \\
& Y_{2}=\delta_{21} Y_{1}+\sum_{j \in G_{2}} \gamma_{2 j} X_{j}+\sum_{l \in G_{C}} \gamma_{C 2 l} X_{l}+e_{2}
\end{aligned}
$$

Here, $Y_{1}$ and $Y_{2}$ are the two standardized phenotypes; $\delta_{12}$ is the causal effect $Y_{2} \rightarrow$ $Y_{1}$ and $\delta_{21}$ is the causal effect $Y_{1} \rightarrow Y_{2} ; X_{i}, X_{j}$ and $X_{l}$ represent the standardized genotype of $i$-th, $j$-th and $l$-th SNP in $G_{1}, G_{2}$ and $G_{c}$ component, respectively; $\gamma_{1 i}, i \in$ $G_{1}$ and $\gamma_{2 j}, j \in G_{2}$ denote the direct effect sizes of phenotype-specific SNPs for $Y_{1}$ and $Y_{2} ; \gamma_{C 1 l}$ and $\gamma_{C 2 l}, l \in G_{C}$ denote the direct effect sizes of pleiotropic SNPs for $Y_{1}$ and $Y_{2}$ respectively; $e_{1}$ and $e_{2}$ are residual effects. Since the SNPs in $G_{0}$ have no direct effects on either phenotype, they are not included. For the direct effect sizes of SNPs, we assume $\gamma_{1 i} \sim N\left(0, \sigma_{1}^{2}\right), \gamma_{2 j} \sim N\left(0, \sigma_{2}^{2}\right)$ and $\left(\begin{array}{l}\gamma_{C 1 l} \\ \gamma_{C 2 l}\end{array}\right) \sim N\left[\left(\begin{array}{l}0 \\ 0\end{array}\right),\left(\begin{array}{cc}\sigma_{C 1}^{2} & \rho_{C 1, C 2} \\ \rho_{C 1, C 2} & \sigma_{C 2}^{2}\end{array}\right)\right]$ for $i \in G_{1}, j \in G_{2}, l \in G_{C}$, where $\sigma_{1}^{2}$ and $\sigma_{2}^{2}$ denote the per-SNP variances of $G_{1}$ and $G_{2}$, and $\sigma_{C 1}^{2}$ and $\sigma_{C 2}^{2}$ the per-SNP variances of $G_{C}$ for $Y_{1}$ and $Y_{2}$ respectively, with covariance $\rho_{C 1, C 2}$. Therefore, the model encompasses both independent pleiotropy $\left(\rho_{C 1, C 2}=0\right)$ and correlated pleiotropy $\left(\rho_{C 1, C 2} \neq 0\right)$.

The above formulae can be expressed in the bivariate matrix form $\boldsymbol{Y}=$ $[\boldsymbol{I}-\boldsymbol{\Delta}]^{-\mathbf{1}} \sum_{\boldsymbol{h}} \sum_{\boldsymbol{k} \in \boldsymbol{h}} \boldsymbol{\Gamma}_{k}^{(h)} X_{\boldsymbol{k}}+\boldsymbol{\varepsilon}$, where $\boldsymbol{Y}$ is a $2 \times 1$ vector of the two standardized phenotypes, $\boldsymbol{I}$ is the $2 \times 2$ identity matrix, $\Delta=\left(\begin{array}{cc}0 & \delta_{12} \\ \delta_{21} & 0\end{array}\right), \Gamma_{k}^{(h)}$ is the direct effect of the $k$-th SNP on the phenotypes depending on its component membership $h$, i.e. $h \in\left(G_{0}, G_{1}, G_{2}, G_{C}\right)$ and $\Gamma_{k}^{\left(G_{0}\right)}=\left(\begin{array}{l}0 \\ 0\end{array}\right), \Gamma_{k}^{\left(G_{1}\right)}=\left(\begin{array}{c}\gamma_{1 k} \\ 0\end{array}\right), \Gamma_{k}^{\left(G_{2}\right)}=\left(\begin{array}{c}0 \\ \gamma_{2 k}\end{array}\right), \Gamma_{k}^{\left(G_{C}\right)}=\left(\begin{array}{l}\gamma_{C 1 k} \\ \gamma_{C 2 k}\end{array}\right)$, $X_{k}$ is the standardized genotype for the $k$-th SNP, $\varepsilon$ is the residual effects of any nonadditive genetic and environmental factors. This expression is similar to that used for reciprocal causal modeling in twin data ${ }^{25}$ and requires that $\left|\delta_{12} \delta_{21}\right|<1$ to ensure that the reciprocal causation between the two phenotypes results in a steady state rather than unlimited growth ${ }^{26}$. In practice, we constrain both $\delta_{12}$ and $\delta_{21}$ to be between -1.0 and 1.0, since both phenotypes are standardized variances 1.0, ensuring the inequality. 
If we define $\boldsymbol{\beta}_{k}^{(h)}=[\boldsymbol{I}-\boldsymbol{\Delta}]^{-\mathbf{1}} \boldsymbol{\Gamma}_{k}^{(h)}$ as a $2 \times 1$ vector of the component-dependent joint effect sizes for the $k$-th SNP, including any effect mediated through one phenotype on the other, then the model can be expressed as $\boldsymbol{Y}=\sum \boldsymbol{\beta}_{k}^{(h)} X_{k}+\boldsymbol{\varepsilon}$. For each phenotype, the marginal effect of SNP $k$ is $\tau_{k}=\sum_{i=1}^{N_{k}^{*}} \beta_{i} \rho_{k i}$, where $N_{k}^{*}$ is the total number of SNPs tagged by the $k$-th SNP, $\rho_{k i}$ is the LD correlation between $k$-th and $i$-th SNP, and $\beta_{i}$ is the joint effect size of the $i$-th SNP tagged by the $k$-th SNP 27,28 , taking account of the reciprocal causations.

\section{Composite likelihood estimator}

We assume that the estimates of the marginal effects of the $k$-th SNP from the GWAS summary statistics have a bivariate normal distribution:

$$
\hat{\boldsymbol{\tau}}_{\boldsymbol{k}}=\left(\begin{array}{c}
\hat{\tau}_{1 k} \\
\hat{\tau}_{2 k}
\end{array}\right) \sim \sum_{\mathbb{N}_{k}} \operatorname{Pr}\left(\mathbb{N}_{k}\right) N\left[\left(\begin{array}{l}
0 \\
0
\end{array}\right),\left(\begin{array}{cc}
\sigma_{\hat{\tau}_{1 k}}^{2} & \rho_{\hat{\tau}_{1 k}, \hat{\tau}_{2 k}} \\
\rho_{\hat{\tau}_{1 k}, \hat{\tau}_{2 k}} & \sigma_{\hat{\tau}_{2 k}}^{2}
\end{array}\right)\right]
$$

In the formula, $\hat{\tau}_{1 k}$ and $\hat{\tau}_{2 k}$ represent the marginal effect size estimates of the $k$-th SNP from GWAS summary statistics of phenotype $Y_{1}$ and $Y_{2}$ respectively. $\mathbb{N}_{k}=$ $\left(N_{k}^{\left(G_{1}\right)}, N_{k}^{\left(G_{2}\right)}, N_{k}^{\left(G_{C}\right)}, N_{k}^{\left(G_{0}\right)}\right)$ are the possible combinations of counts of SNPs in LD with SNP $k$ that belong to the four SNP components. The probability of each combination, $\operatorname{Pr}\left(\mathbb{N}_{k}\right)$, can be calculated based on the multinomial distribution with total counts $N_{k}^{*}=\sum_{h} N_{k}^{(h)}$ over $h \in\left(G_{0}, G_{1}, G_{2}, G_{C}\right)$, where $N_{k}^{(h)}$ is a latent variable denoting the number of $h$-component SNPs tagged by the $k$-th SNP. $\sigma_{\hat{\tau}_{1 k}}^{2}, \sigma_{\hat{\tau}_{2 k}}^{2}$ and $\rho_{\hat{\tau}_{1 k}, \hat{\tau}_{2 k}}$ can be derived from the relationships between the marginal and joint regression coefficients in mixture form (see Supplementary Note):

$$
\begin{aligned}
& \sigma_{\widehat{\tau}_{1 k}}^{2} \approx \frac{\sigma_{1}^{2}}{\left(1-\delta_{12} \delta_{21}\right)^{2}} \frac{N_{k}^{\left(G_{1}\right)}}{N_{k}^{*}} \ell_{k}+\frac{\delta_{12}^{2} \sigma_{2}^{2}}{\left(1-\delta_{12} \delta_{21}\right)^{2}} \frac{N_{k}^{\left(G_{2}\right)}}{N_{k}^{*}} \ell_{k}+\frac{\left.\left[\sigma_{C 1}^{2}+\delta_{12}^{2} \sigma_{C 2}^{2}+2 \delta_{12} \rho \rho_{C 1, C 2}\right)\right]}{\left(1-\delta_{12} \delta_{21}\right)^{2}} \frac{N_{k}^{(G C)}}{N_{k}^{*}} \ell_{k}+a_{1}+1 / n_{1} \\
& \sigma_{\hat{\tau}_{2 k}}^{2} \approx \frac{\delta_{12}^{2} \sigma_{1}^{2}}{\left(1-\delta_{12} \delta_{21}\right)^{2}} \frac{N_{k}^{\left(G_{1}\right)}}{N_{k}^{*}} \ell_{k}+\frac{\sigma_{2}^{2}}{\left(1-\delta_{12} \delta_{21}\right)^{2}} \frac{N_{k}^{\left(G_{2}\right)}}{N_{k}^{*}} \ell_{k}+\frac{\left[\sigma_{C 2}^{2}+\delta_{12}^{2} \sigma_{C 1}^{2}+2 \delta_{21} \rho_{C 1}, C_{2}\right]}{\left(1-\delta_{12} \delta_{21}\right)^{2}} \frac{N_{k}^{\left(G_{C}\right)}}{N_{k}^{*}} \ell_{k}+a_{2}+1 / n_{2} \\
& \rho_{\hat{\tau}_{1 k}, \hat{\tau}_{2 k}} \approx \frac{\delta_{21} \sigma_{1}^{2}}{\left(1-\delta_{12} \delta_{21}\right)^{2}} \frac{N_{k}^{\left(G_{1}\right)}}{N_{k}^{*}} \ell_{k}+\frac{\delta_{12} \sigma_{2}^{2}}{\left(1-\delta_{12} \delta_{21}\right)^{2}} \frac{N_{k}^{\left(G_{2}\right)}}{N_{k}^{*}} \ell_{k}+\frac{\left[\delta_{21} \sigma_{C 1}^{2}+\delta_{12} \sigma_{C 2}^{2}+\left(1+\delta_{12} \delta_{21}\right) \rho_{\left.C 1, C_{2}\right]}\right.}{\left(1-\delta_{12} \delta_{21}\right)^{2}} \frac{N_{k}^{\left(G_{C}\right)}}{N_{k}^{*}} \ell_{k}+\rho_{0}
\end{aligned}
$$

where $\ell_{k}$ is the LD score for the $k$-th SNP; $a_{1}$ and $a_{2}$ are additional inflation factors accounting for systematic bias in variance estimates (e.g., due to population stratification) for phenotype $Y_{1}$ and $Y_{2}$ respectively ${ }^{28} ; \rho_{0}$ is a factor accounting for bias in the covariance estimates (e.g. due to sample overlap); $n_{1}$ and $n_{2}$ are the sample sizes for the two GWAS of the two phenotypes. In this way, the partitioned genetic variances, LD conditions as well as the reciprocal causal effects are reflected in the assumed bivariate distribution.

The likelihood for the summary-statistic of the $k$-th SNP is then: $L\left(\boldsymbol{\theta} ; \hat{\boldsymbol{\tau}}_{\boldsymbol{k}}\right)=$ $p\left(\hat{\boldsymbol{\tau}}_{\boldsymbol{k}} \mid \boldsymbol{\theta}\right)=\sum_{\mathbb{N}_{k}} \operatorname{Pr}\left(\mathbb{N}_{k}\right) f\left(\hat{\tau}_{1 k}, \hat{\tau}_{2 k}\right)$, where $f\left(\hat{\tau}_{1 k}, \hat{\tau}_{2 k}\right)$ is the density function of bivariate normal distribution with parameters $\boldsymbol{\theta}=$ $\left(\pi_{1}, \pi_{2}, \pi_{c}, \sigma_{1}^{2}, \sigma_{2}^{2}, \sigma_{C 1}^{2}, \sigma_{C 2}^{2}, \rho_{C 1, C 2}, \delta_{12}, \delta_{21}, a_{1}, a_{2}, \rho_{0}\right)$. Thus, the composite loglikelihood function is in the form:

$$
C L(\boldsymbol{\theta} ; \hat{\boldsymbol{\tau}})=\sum_{k=1}^{K} \log L\left(\boldsymbol{\theta} ; \hat{\boldsymbol{\tau}}_{\boldsymbol{k}}\right)=\sum_{k=1}^{K} \log \left[\sum_{\mathbb{N}_{k}} \operatorname{Pr}\left(\mathbb{N}_{k}\right) f\left(\hat{\tau}_{1 k}, \hat{\tau}_{2 k}\right)\right]
$$


Thus, the maximum composite likelihood estimator is given by

$$
\widehat{\boldsymbol{\theta}}=\underset{\boldsymbol{\theta}}{\operatorname{argmax}} C L(\boldsymbol{\theta} ; \hat{\boldsymbol{\tau}})
$$

\section{Parameter estimation and testing}

The reciprocal causal paths ( $\delta_{12}$ and $\delta_{21}$ ), together with nuisance parameters, are estimated by maximizing the likelihood with an expectation-maximization (EM) algorithm. For each M-step in the EM, parameters for mixing proportions $\left(\pi_{1}, \pi_{2}\right.$ and $\pi_{c}$ ) are estimated according to the closed form, while the remaining parameters (i.e. $\left.\sigma_{1}^{2}, \sigma_{2}^{2}, \sigma_{C 1}^{2}, \sigma_{C 2}^{2}, \rho_{C 1, C 2}, \delta_{21}, \delta_{21}, a_{1}, a_{2}, \rho_{0}\right)$ are estimated by Nelder-Mead optimization (see Supplementary Note).

The standard errors of each parameter are calculated using a sandwich variance estimator adapted from Zhang et al ${ }^{28}$, since sandwich estimators can provide valid variance estimation and tolerate the possible misspecification of the model. Briefly, if we define $l_{k}(\boldsymbol{\theta})=\log L\left(\boldsymbol{\theta} ; \hat{\boldsymbol{\tau}}_{\boldsymbol{k}}\right)$ then the score function could be written as in the form: $U(\boldsymbol{\theta})=\frac{\partial C L\left(\boldsymbol{\theta} ; \hat{\boldsymbol{\tau}}_{\boldsymbol{k}}\right)}{\partial \boldsymbol{\theta}}=\sum_{k=1}^{K} \frac{\partial \log L\left(\boldsymbol{\theta} ; \hat{\boldsymbol{\tau}}_{\boldsymbol{k}}\right)}{\partial \boldsymbol{\theta}}=\sum_{k=1}^{K} \frac{\partial l_{k}(\boldsymbol{\theta})}{\partial \boldsymbol{\theta}}=\sum_{k=1}^{K} U_{k}(\boldsymbol{\theta})$, where $U_{k}(\boldsymbol{\theta})$ is the score vector for the $k$-th SNP. Then the sandwich form of variance-covariance matrix for the estimate $\widehat{\boldsymbol{\theta}}$ is $\operatorname{var}(\widehat{\boldsymbol{\theta}})=I^{-1}(\boldsymbol{\theta}) J(\boldsymbol{\theta}) I^{-1}(\boldsymbol{\theta})$, which can be estimated by plugging in the estimated parameter values $\widehat{\boldsymbol{\theta}}$ in lieu of $\boldsymbol{\theta}$. Thus, we can get $\hat{I}(\widehat{\boldsymbol{\theta}})=$ $-\left.\sum_{k=1}^{K} \frac{\partial l_{k}^{2}(\boldsymbol{\theta})}{\partial \boldsymbol{\theta} \partial \boldsymbol{\theta}^{T}}\right|_{\widehat{\boldsymbol{\theta}}}$ and $\hat{J}(\widehat{\boldsymbol{\theta}})=\sum_{k=1}^{K} U_{k}(\widehat{\boldsymbol{\theta}}) \bar{U}_{k}^{T}(\widehat{\boldsymbol{\theta}})$, where $\bar{U}_{k}^{T}(\widehat{\boldsymbol{\theta}})=\sum_{k^{\prime} \in \mathbb{N}_{k}} U_{k^{\prime}}(\widehat{\boldsymbol{\theta}})$ is the sum of likelihood scores for all SNPs tagged by the $k$-th SNP. In practice, we used the symmetric derivative to obtain the derivatives for parameter estimates (see Supplementary Note). To determine the significance of estimate for parameters of interest, estimates and the corresponding standard errors are converted to $\chi^{2}$ statistics.

\section{Sub-models and model averaging}

To increase the robustness of $\mathrm{MRCl}$ in real situations, we performed model fitting not only to the full model (referred to as $s_{1,2, C}$ model) but also to four sub-models, each of which has one or two absent SNP components: (I) the $s_{2, C}$ model excludes $G_{1}$ SNPs; (II) the $s_{1, C}$ model excludes $G_{2}$ SNPs; (III) the $s_{C}$ model excludes both $G_{1}$ and $G_{2}$ SNPs; (IV) the $s_{1,2}$ model excludes $G_{C}$ SNPs. For each sub-model, the parameters are estimated using the same composite likelihood estimator as above, only that the parameters of the corresponding absent components are set as zero during the EM process and are thus excluded in variance calculation. Accordingly, we define the complete model set $\boldsymbol{S}=\left(s_{1,2, C}, s_{2, C}, s_{1, C}, s_{1,2}, s_{C}\right)$.

After estimating all five models, we calculated weighted averages of the parameter estimates of these models to obtain an "averaged" model, and calculated its composite likelihood. The averaged estimate for the $j$-th parameter can be written as $\hat{\theta}_{j, m a}=\sum_{S}^{S} \widehat{w}_{s} \hat{\theta}_{j, s}$, where $\widehat{w}_{S}$ is the weight for the $s$-th model and $\hat{\theta}_{j, s}$ is the estimate of the $j$-th parameter in the $s$-th model. $\hat{\theta}_{j, s}$ is zero if the $j$-th parameter is not included in the $s$-th model. Then, variance of the $j$-th averaged parameter can be 
calculated as $\operatorname{var}\left(\hat{\theta}_{j, m a}\right)=\left(\sum_{s}^{S} \widehat{w}_{s} \sqrt{\operatorname{var}\left(\hat{\theta}_{j, s}\right)+\left(\hat{\theta}_{j, s}-\hat{\theta}_{j, m a}\right)^{2}}\right)^{2}$, where $\operatorname{var}\left(\hat{\theta}_{j, s}\right)$ is the variance of the $j$-th parameter in the $s$-th model and is defined as zero if the $j$ th parameter is not included in the $s$-th model ${ }^{29}$. The composite likelihood is then maximized over the weights for the five models, where the initial weights for the optimization are based on a modified Akaike information criterion (AIC) for composite likelihood $^{30}$, for the five models (see Supplementary Note). Finally, we compared the composite likelihood obtained in this way through model averaging to that obtained by applying the EM algorithm to the $s_{1,2, C}$ model, and chose the parameter estimates with the higher composite likelihood as the final parameter estimates.

\section{Genetic correlation calculation}

Based on our reciprocal joint model, genetic correlation $\left(r_{g}\right)$ between the two phenotypes is calculated as:

$$
r_{g}=\frac{\pi_{1} \delta_{21} \sigma_{1}^{2}+\pi_{2} \delta_{12} \sigma_{2}^{2}+\pi_{C}\left[\delta_{21} \sigma_{C 1}^{2}+\delta_{12} \sigma_{C 2}^{2}+\left(1+\delta_{12} \delta_{21}\right) \rho_{C 1, C 2}\right]}{\sqrt{\left.\left(\pi_{1} \sigma_{1}^{2}+\pi_{2} \delta_{12}^{2} \sigma_{2}^{2}+\pi_{C}\left[\sigma_{C 1}^{2}+\delta_{12}^{2} \sigma_{C 2}^{2}\right)\right]\right) \cdot\left(\pi_{1} \delta_{21}^{2} \sigma_{1}^{2}+\pi_{2} \sigma_{2}^{2}+\pi_{C}\left[\delta_{21}^{2} \sigma_{C 1}^{2}+\sigma_{C 2}^{2}\right]\right)}}
$$

The derivation of $r_{g}$ and the corresponding variance calculation can be found in Supplementary Note.

\section{Simulation data}

The simulation data were generated using imputed genotype of 50,000 individuals randomly extracted from UK Biobank white British subjects. We only selected common SNPs (i.e. MAF $\geq 0.05$ ) that are available in the HapMap 3 reference panel resulting in a total of about 0.68 million SNPs. Sample sizes for $Y_{1}$ and $Y_{2}$ were both 50,000 , with complete sample overlap. Simulation data in this paper are based on these $100 \%$ overlapping 50,000 samples, unless otherwise specified.

In each simulation scenario, we first defined values of $\pi_{1}, \pi_{2}, \pi_{c}, \sigma_{1}^{2}, \sigma_{2}^{2}, \sigma_{C 1}^{2}, \sigma_{C 2}^{2}, \rho_{C 1, C 2}, \delta_{12}, \delta_{21}$ for the scenario. We randomly chose causal SNPs from the set of all selected common SNPs according to the assigned mixing proportions for the four SNP components. For trait-specific SNPs, the direct effect sizes for causal SNPs were assigned based on $\gamma_{1 i} \sim N\left(0, \sigma_{1}^{2}\right)$ and $\gamma_{2 j} \sim N\left(0, \sigma_{2}^{2}\right)$ with $i \in G_{1}$ and $j \in G_{2}$. For pleiotropic SNPs, the direct effect sizes for causal SNPs were assigned based on $\left(\begin{array}{l}\gamma_{C 1 l} \\ \gamma_{C 2 l}\end{array}\right) \sim N\left[\left(\begin{array}{l}0 \\ 0\end{array}\right),\left(\begin{array}{cc}\sigma_{C 1}^{2} & \rho_{C 1, C 2} \\ \rho_{C 1, C 2} & \sigma_{C 2}^{2}\end{array}\right)\right]$ with $l \in G_{C}$. Next, we converted the direct effect sizes to the component-dependent joint effect sizes using $\boldsymbol{\beta}_{k}^{(h)}=[\boldsymbol{I}-\boldsymbol{\Delta}]^{-\mathbf{1}} \boldsymbol{\Gamma}_{k}^{(h)}$ to take account of reciprocal causation. Then we simulated the two phenotypes $\left(Y_{1}\right.$ and $Y_{2}$ ) with these joint effect sizes using GCTA software ${ }^{31}$ and performed the association test using PLINK ${ }^{32}$ to obtain the summary-level results. Finally, we repeated the simulations 100 times for each scenario.

We generated data under three causation scenarios: "bi-directional causation" when there are causal effects in both directions, "uni-directional causation" when there is causal effect in only one direction, and "null causation" when there is no causal effect in either direction. We considered low and high polygenicity scenarios. For low 
polygenicity scenarios, we performed two sets of simulation studies: (I) independent pleiotropy $\left(\pi_{1}=\pi_{2}=\pi_{c}=1 \times 10^{-4}\right.$ and $\left.\rho_{C 1, C 2}=0.0\right)$; and (II) correlated pleiotropy $\left(\pi_{1}=\pi_{2}=\pi_{c}=1 \times 10^{-4}\right.$ and $\left.\rho_{C 1, C 2}=0.1\right)$ (Figure 1b). For high polygenicity scenarios, we set $\pi_{1}=\pi_{2}=\pi_{c}=1 \times 10^{-3}$ and performed six simulation studies under correlated pleiotropy considering bi-directional, unidirectional and null causations. We also additionally simulated a high polygenicity scenario in which the two phenotypes had different mixing proportions and genetic variances. For simulation studies with different sample sizes, we set $\pi_{1}=\pi_{2}=$ $\pi_{c}=1 \times 10^{-3}$ and decreased the sample sizes to 20,000 under correlated pleiotropy scenarios. We generated simulated data under sub-models with high polygenicity and correlated pleiotropy, and for bi-directional, uni-directional and null causations.

For simulations involving binary phenotypes, we assumed $Y_{1}$ to be the continuous phenotype and $Y_{2}$ to be the binary phenotypes. For $Y_{1}$ we used the above method (with 50,000 individuals) to obtain GWAS summary data. For $Y_{2}$, we generated the joint effect sizes on the liability scale, then took 45,000 individuals to simulate a case-control study with disease prevalence 0.05 and case:control ratio $1: 2$, using GCTA. We then ran logistic regression analysis using PLINK to obtain the GWAS summary statistics for $Y_{2}$. Since the parameters in our model are on the liability scale, we converted the summary-level estimates of odds ratios to the liability scale effect size through approximation ${ }^{33,34}$ (see Supplementary Note).

Detailed parameter settings and representative bivariate scatterplots for these simulations can be found in Supplementary Table S1 and Figure S1.

\section{Existing MR methods and LD score regression}

We also ran several IV-based MR methods on the simulated data: Egger regression, Weighted Median, Weighted Mode, Inverse-Variance Weighted (IVW) (from the TwoSampleMR package ${ }^{3}$ ), MRMix ${ }^{12}$ and MR-PRESSO ${ }^{11}$. We tested three SNP selection methods: (a) use valid IVs, i.e. all the exposure-specific true causal SNPS assigned in the simulation as IVs; (b) use significant exposure-associated SNPs ( $p$ value $<5 \times 10^{-8}$ ) but exclude potential outcome-associated SNPs by setting $p$ value $>5 \times 10^{-5}$ in outcome GWAS; (c) use significant exposure-associated SNPs regardless of their association with outcome. For SNPs selected in (b) and (c), we further performed clumping $\left(r^{2}<0.01\right)$ to obtain the independent IVs for MR analysis. Additionally, we also compared with CAUSE ${ }^{13}$, a recent method using genome-wide summary statistics. The $p$-value thresholds for CAUSE and MRMix were set as $1 \times 10^{-3}$ and $5 \times 10^{-8}$, respectively. To calculate the genetic correlation using LD Score regression ${ }^{1}$, we followed the online tutorial and used the default settings.

\section{Real data processing}

We followed steps similar to those implemented by Zhang et $a^{28}$ to preprocess the public GWAS summary data. Briefly, SNPs were excluded if MAF was less than 5\%; or if the imputation INFO score was low (INFO < 0.9); or if the available sample size was less than 0.67 of the 90th percentile of the available sample sizes for all SNPs; or if it 
was located within the major histocompatibility complex (MHC) region. The summary statistics of remaining SNPs were merged with 1000 Genome reference SNPs to obtain their corresponding LD scores.

\section{Code availability}

The $\mathrm{MRCl}$ method can be found at https://github.com/zpliu/MRCI. Detailed results of all real data estimation can be visualized through the website:

https://triangularcell.shinyapps.io/MRCl_Estimate_for_CommonDiseases.

\section{Acknowledgments}

We thank the consortia for sharing the GWAS summary statistics. We thank Dr. Robert Porsch for critical advice on the model. The simulation studies were conducted using genotype data from the UK Biobank Resource accessed under Application Number 28732. The computations were performed using research computing facilities offered by Information Technology Services, The University of Hong Kong and the High Performance Computing Facility of Bioinformatics Core, Centre for PanorOmic Sciences (CPOS), LKS Faculty of Medicine, The University of Hong Kong. This work was supported by Hong Kong Research Grants Council Collaborative Research Grant C7044-19G and Hong Kong Innovation and Technology Bureau funding for the State Key Laboratory of Brain and Cognitive Sciences.

\section{Author contributions}

P.C.S., Y.D.Z., Z.L. and Y.Q. conceived and designed the model and contributed to the interpretation of the results. Z.L. and Y.Q. designed the algorithm, implemented the software and conducted analysis of simulation and real data. T.S.H.M. prepared the genotype data for simulation. T.W. contributed to statistical derivation. Y.D.Z. and M.L. prepared LD data and developed the software. L.B. and J.D.T. contributed to interpretation of real data analysis. P.C.S., Z.L. and Y.Q. contributed to writing the manuscript. T.W., J.D.T., L.B., M.L. and Y.D.Z. contributed to critical revision of the manuscript.

\section{Competing interests}

The authors declare no competing interests. 


\section{Figure Legends}

Figure 1. Schematic diagram of $\mathbf{M R C l}$. a, $Y_{1}$ and $Y_{2}$ represent a pair of phenotypes. Genotypes can be divided into four components: $Y_{1}$-specific causal SNPs $\left(G_{1}\right), Y_{2^{-}}$ specific causal SNPs $\left(G_{2}\right)$, pleiotropic causal SNPs $\left(G_{C}\right)$ and null SNPs $\left(G_{0}\right.$, not shown). Lines connecting these genotypes represent the LD correlation between SNPs. Arrow lines from genotype to phenotype represent the direct effect of corresponding SNPS $\left(\gamma_{1}, \gamma_{2}, \gamma_{C 1}\right.$ and $\left.\gamma_{C 2}\right)$ on the phenotypes. The covariance $\left(\rho_{C 1, C 2}\right)$ between $\gamma_{C 1}$ and $\gamma_{C 2}$ is allowed. Arrow lines between the two phenotypes represent the reciprocal causal paths $\left(\delta_{12}\right.$ and $\left.\delta_{21}\right)$. Non-additive genetic effects on phenotypes are represented by $e_{1}$ and $e_{2}$. In real situations, one or two components in the model could be absent and our method could handle these sub-model scenarios in a robust way. $\mathbf{b}$, illustration of several representative simulation scenarios. $x$-axis and $y$-axis show the standardized effect size estimates for GWAS $Y_{1}$ and $Y_{2}$ respectively. Green, red, orange and grey points represent pleiotropic, $Y_{1}$-specific, $Y_{2}$-specific and null SNPs in the simulation respectively. For null causation, $\delta_{12}=\delta_{21}=0.0$; for unidirectional causation, $\delta_{12}=0.1, \delta_{21}=0.0$; for bi-directional causation, $\delta_{12}=0.1$, $\delta_{21}=0.05$. For independent pleiotropy, $\rho_{C 1, C 2}=0.0$; for correlated pleiotropic effects, $\rho_{C 1, C 2}=0.1$. In these plots, the mixing proportions for non-null components were set as $\pi_{1}=\pi_{2}=\pi_{c}=1 \times 10^{-4}$, and the heritability contributed by $Y_{1}$ specific, $Y_{2}$-specific and pleiotropic SNPs were set as $0.3,0.3$ and 0.1 respectively. (see Supplementary Figure S1 for other simulated scenarios)

Figure 2. Comparison of estimates for the reciprocal causal effect by our method and instrumental variable (IV)-based MR methods in simulations with correlated pleiotropy. a, estimates in null causation scenarios $\left(\delta_{12}=\delta_{21}=0.0\right)$. b, estimates in uni-directional causation scenarios $\left(\delta_{12}=0.1\right.$ and $\left.\delta_{21}=0.0\right)$. c, estimates in bidirectional causation scenarios ( $\delta_{12}=0.1$ and $\delta_{21}=0.05$ ). d, rejection rates of null hypothesis in bi-directional, uni-directional and null scenarios. In plots $\mathbf{a}, \mathbf{b}$ and $\mathbf{c}$, our method took genome-scale SNPs for estimation and produced nearly unbiased estimates in different scenarios. For MR methods, IVs were selected in three ways:

(1) use the exposure-specific true causal SNPs in the simulation as IVs; (2) use exposure-associated SNPs ( $p$-value $<5 \times 10^{-8}$ ) after clumping but exclude potential outcome-associated SNPs (defined as p-value $<5 \times 10^{-5}$ with outcome); (3) use significant exposure-associated SNPs after clumping regardless of their association with outcome. In plot $\mathbf{d}$, the exclusion criteria were applied to IV-based MR methods and our method shows well-controlled Type I error rates and adequate power. In these plots, the true values of $\delta_{12}$ and $\delta_{21}$ are indicated by up- and down-pointing triangles, respectively; $\rho_{C 1, C 2}$ for the correlated pleiotropy is 0.1 ; the mixing proportions for non-null components are $\pi_{1}=\pi_{2}=\pi_{c}=1 \times 10^{-4}$; the heritability contributed by $Y_{1}$-specific, $Y_{2}$-specific and pleiotropic SNPs are $0.3,0.3$ and 0.1 respectively. (Note: The selection of exposure-specific true causal SNPs was not applied to MRMix due to its assumption of a normal-mixture distribution)

Figure 3. Estimation after model averaging. a, final estimates of causal effects under bi-directional, uni-directional and null causations in four scenarios $\left(s_{1,2, C}, s_{2, C}, s_{1,2}\right.$ and $\left.s_{C}\right)$. When the exposure-specific SNPs exist, the final estimates after model averaging still produced nearly unbiased estimates in simulated scenarios. For null 
causation (grey), $\delta_{12}=\delta_{21}=0.0$; for uni-directional causation (blue), $\delta_{12}=0.1$ and $\delta_{21}=0.0$; for bi-directional causation (purple), $\delta_{12}=0.1$ and $\delta_{21}=0.05$. The true causation values of $\delta_{12}$ and $\delta_{21}$ are indicated by up- and down-pointing triangles, respectively. $\mathbf{b}$, rejection rate of null hypothesis of the final $\delta_{12}$ and $\delta_{21}$ estimates after model averaging in different scenarios. For zero-effect causal direction, the Type I error rates are well-controlled; for non-zero-effect causal direction, the presence of the exposure-specific SNPs shows reasonably good power, and the absence of the exposure-specific SNPs shows conservative power of estimation. In the simulations, the mixing proportion of the present component was $1 \times 10^{-3}$; the pleiotropic effects were correlated $\left(\rho_{C 1, C 2}=0.1\right)$; the heritability contributed by $Y_{1^{-}}$specific, $Y_{2}$-specific and pleiotropic SNPs (if present in the sub-model scenario) were $0.3,0.3$ and 0.1 , respectively.

Figure 4. Estimation comparison with CAUSE and MRMix. a, estimates from our method (green), CAUSE (yellow) and MRMix (blue) under bi-directional, unidirectional and null causations in four scenarios $\left(s_{1,2, C}, s_{2, C}, s_{1,2}\right.$ and $\left.s_{C}\right)$. CAUSE and MRMix produced severely over-estimated causal effects when exposure-specific SNPs are absent in the sub-model $s_{2, C}$ and $s_{C}$. For null causation, $\delta_{12}=\delta_{21}=0.0$; for uni-directional causation, $\delta_{12}=0.1$ and $\delta_{21}=0.0$; for bi-directional causation, $\delta_{12}=0.1$ and $\delta_{21}=0.05$. The true causation values of $\delta_{12}$ and $\delta_{21}$ are indicated by up- and down-pointing triangles, respectively. $\mathbf{b}$, rejection rate of null hypothesis for $\delta_{12}$ and $\delta_{21}$ estimates from our method (green), CAUSE (orange) and MRMix (blue) in different simulated scenarios. CAUSE and MRMix produced inflated Type I error rates for the causal direction where exposure-specific SNPs were absent $\left(s_{2, C}\right.$ and $s_{C}$ ). c, estimates of causal effects and rejection rate of null hypothesis from our method (green), CAUSE (orange) and MRMix (blue) under bi-directional, unidirectional and null causations in $s_{1,2, C}$ scenario with small sample sizes $(20,000$ individuals). Decreased GWAS power led to over-estimates for CAUSE and larger estimation variance for MRMix. Our method produced nearly unbiased correct Type I error rates. In these results, the estimates of our method came from the final estimates after model averaging; P-value thresholds for CAUSE and MRMix were $1 \times 10^{-3}$ and $5 \times 10^{-8}$, respectively. In the simulations, the mixing proportion of the present component was $1 \times 10^{-3}$; the pleiotropic effects were correlated $\left(\rho_{C 1, C 2}=0.1\right)$; the heritability contributed by $Y_{1}$-specific, $Y_{2}$-specific and pleiotropic SNPs (if present in the sub-model scenario) were $0.3,0.3$ and 0.1 , respectively.

Figure 5. Summary of estimates for three common diseases and sixteen risk factors. In the figure, the upper triangle in a box represents the causal direction from the disorder to the risk factor, and the lower triangle represents the reverse causal direction. Darker color represents stronger causation estimates. Stars in the triangle represent different significance levels of the estimation. Abbreviations: CAD coronary artery disease; IS - any ischemic stroke; T2D - type 2 diabetes; BirthWeight - birth weight; BMI - body mass index; BodyFat - body fat percentage; FastGluc fasting glucose level; FastInsulin - fasting insulin level; HDL - high-density lipoprotein cholesterol level; LDL - low-density lipoprotein cholesterol level; Triglycerides triglyceride level; CRP - C-reactive protein level; CigPerDay - cigarettes per day; 
DrinksPerWeek - drinks per week; pulsePressure - pulse pressure; dBP - diastolic blood pressure; SBP - systolic blood pressure; MDD - major depressive disorder. 
Table

\begin{tabular}{|c|c|c|c|c|c|c|}
\hline Traits & $\begin{array}{c}\text { Total } \\
\text { reported } \\
\text { sample size }\end{array}$ & $\begin{array}{l}\text { Number of } \\
\text { reported } \\
\text { cases }\end{array}$ & $\begin{array}{l}\text { Number of } \\
\text { reported } \\
\text { controls }\end{array}$ & $\begin{array}{l}\text { Number of } \\
\text { independent } \\
\text { significant } \\
\text { SNPs/loci }\end{array}$ & $\begin{array}{l}\text { Variance } \\
\text { explained by } \\
\text { significant } \\
\text { SNPs/loci }\end{array}$ & Publication \\
\hline CAD & 148,172 & 10,801 & 137,371 & 66 & $21.2 \%$ & $\begin{array}{c}\text { Nelson et al }{ }^{35} \text {. } \\
2017 .\end{array}$ \\
\hline IS & 440,328 & 34,217 & 406,111 & 2 & $0.6 \%-1.8 \%$ & $\begin{array}{c}\text { Malik et al }{ }^{36} \text {. } \\
2018 .\end{array}$ \\
\hline $\mathrm{T} 2 \mathrm{D}$ & 898,130 & 74,124 & 824,006 & 243 & $18.0 \% \$$ & $\begin{array}{l}\text { Mahajan et } \\
\mathrm{al}^{37} .2018 .\end{array}$ \\
\hline BirthWeight & 298,142 & - & - & 190 & $7.0 \%$ & $\begin{array}{c}\text { Warrington et } \\
\mathrm{al}^{38} .2019 .\end{array}$ \\
\hline BMI & 681,275 & - & - & 941 & $6.0 \%$ & $\begin{array}{c}\text { Yengo et al }{ }^{39} \text {. } \\
2018 .\end{array}$ \\
\hline BodyFat & 89,297 & - & - & 7 & $0.6 \%$ & Lu et al40. 2016. \\
\hline Height & 693,529 & - & - & 3,290 & $24.6 \%$ & $\begin{array}{c}\text { Yengo et al }{ }^{39} \text {. } \\
2018 .\end{array}$ \\
\hline FastGluc & $140,595^{\&}$ & - & - & $7^{*}$ & - & $\begin{array}{c}\text { Lagou et al }{ }^{41} \text {. } \\
2021 .\end{array}$ \\
\hline Fastlnsulin & $98,210^{\&}$ & - & - & $1^{*}$ & - & $\begin{array}{c}\text { Lagou et al }{ }^{41} \text {. } \\
2021 .\end{array}$ \\
\hline HDL & 188,577 & - & - & 70 & $1.6 \%$ & $\begin{array}{c}\text { Willer et al42. } \\
2013 .\end{array}$ \\
\hline LDL & 188,577 & - & - & 57 & $2.4 \%$ & $\begin{array}{c}\text { Willer et al42. } \\
2013 .\end{array}$ \\
\hline Triglycerides & 188,577 & - & - & 37 & $2.1 \%$ & $\begin{array}{c}\text { Willer et al42. } \\
2013 .\end{array}$ \\
\hline CRP & 418,642 & - & - & 526 & $13.0 \%$ & $\begin{array}{c}\text { Han et } \mathrm{al}^{43} \text {. } \\
2020 .\end{array}$ \\
\hline CigPerDay & 337,334 & - & - & 55 & 1.1\% & $\begin{array}{c}\text { Liu et al }{ }^{44} \text {. } \\
2019 .\end{array}$ \\
\hline DrinksPerWeek & 941,280 & - & - & 99 & $\sim 0.2 \%$ & $\begin{array}{c}\text { Liu et al }{ }^{44} \text {. } \\
2019 .\end{array}$ \\
\hline pulsePressure & 757,601 & - & - & $143 / 62^{\#}$ & $2.6 \%$ & $\begin{array}{c}\text { Evangelou et } \\
\mathrm{al}^{45} .2018 \text {. }\end{array}$ \\
\hline$d B P$ & 757,601 & - & - & $130 / 120^{\#}$ & $4.5 \%$ & $\begin{array}{c}\text { Evangelou et } \\
\mathrm{al}^{45} .2018 \text {. }\end{array}$ \\
\hline sBP & 757,601 & - & - & $183 / 81^{\#}$ & $4.8 \%$ & $\begin{array}{c}\text { Evangelou et } \\
\mathrm{al}^{45} .2018 .\end{array}$ \\
\hline MDD & 500,199 & 170,756 & 329,443 & $102^{\wedge}$ & $1.5 \% \sim 3.2 \%$ & $\begin{array}{c}\text { Howard et al }{ }^{46} \text {. } \\
2019 .\end{array}$ \\
\hline
\end{tabular}

Table 1. Descriptive summary statistics of included traits. CAD - coronary artery disease; IS - ischemic stroke; T2D - type 2 diabetes; BirthWeight - birth weight; BMI - body mass index; BodyFat - body fat percentage; FastGluc - fasting glucose level; FastInsulin - fasting insulin level; HDL - high-density lipoprotein cholesterol level; LDL - low-density lipoprotein cholesterol level; Triglycerides - triglyceride level; CRP - Creactive protein level; CigPerDay - cigarettes per day; DrinksPerWeek - drinks per week; pulsePressure - pulse pressure; $\mathrm{dBP}$ - diastolic blood pressure; sBP - systolic blood pressure; MDD - major depressive disorder. \& Maximum number of samples used for meta-analysis, not only European population. * Novel discoveries in this

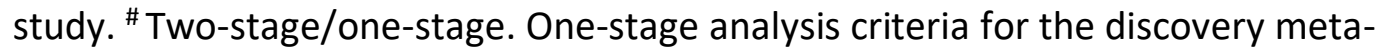
analysis $\left(757,601\right.$ samples): $p$-value $<5 \times 10^{-9}$. One-stage analysis criteria for UKB $(458,577$ samples): $p$-value $<0.01$. One-stage analysis criteria for $\operatorname{ICBP}(299,024$ 
samples): $p$-value $<0.01$ and concordant direction of effect between UKB and ICBP. Two-stage analysis critieria: genome-wide significance in the combined metaanalysis, $p$-value $<0.01$ in the replication meta-analysis and concordant direction of effect. ^ For all meta-analysis, not specific to European populations. \$ Trait variance explained by all GWAS SNPs/loci. 


\section{References}

1. Bulik-Sullivan, B. et al. An atlas of genetic correlations across human diseases and traits. Nat Genet 47, 1236-41 (2015).

2. O'Connor, L.J. \& Price, A.L. Distinguishing genetic correlation from causation across 52 diseases and complex traits. Nature Genetics (2018).

3. Hemani, G. et al. The MR-Base platform supports systematic causal inference across the human phenome. Elife 7(2018).

4. Burgess, S., Butterworth, A. \& Thompson, S.G. Mendelian randomization analysis with multiple genetic variants using summarized data. Genet Epidemiol 37, 658-65 (2013).

5. Davies, N.M., Holmes, M.V. \& Davey Smith, G. Reading Mendelian randomisation studies: a guide, glossary, and checklist for clinicians. BMJ 362, k601 (2018).

6. Davey Smith, G. \& Hemani, G. Mendelian randomization: genetic anchors for causal inference in epidemiological studies. Hum Mol Genet 23, R89-98 (2014).

7. Bowden, J., Davey Smith, G., Haycock, P.C. \& Burgess, S. Consistent Estimation in Mendelian Randomization with Some Invalid Instruments Using a Weighted Median Estimator. Genet Epidemiol 40, 304-14 (2016).

8. Hartwig, F.P., Davey Smith, G. \& Bowden, J. Robust inference in summary data Mendelian randomization via the zero modal pleiotropy assumption. Int J Epidemiol 46, 1985-1998 (2017).

9. Zheng, J. et al. The Effect of Plasma Lipids and Lipid-Lowering Interventions on Bone Mineral Density: A Mendelian Randomization Study. J Bone Miner Res 35, 1224-1235 (2020).

10. Bowden, J., Davey Smith, G. \& Burgess, S. Mendelian randomization with invalid instruments: effect estimation and bias detection through Egger regression. Int J Epidemiol 44, 512-25 (2015).

11. Verbanck, M., Chen, C.Y., Neale, B. \& Do, R. Detection of widespread horizontal pleiotropy in causal relationships inferred from Mendelian randomization between complex traits and diseases. Nat Genet 50, 693-698 (2018).

12. Qi, G. \& Chatterjee, N. Mendelian randomization analysis using mixture models for robust and efficient estimation of causal effects. Nat Commun 10, 1941 (2019).

13. Morrison, J., Knoblauch, N., Marcus, J.H., Stephens, M. \& He, X. Mendelian randomization accounting for correlated and uncorrelated pleiotropic effects using genome-wide summary statistics. Nature Genetics (2020).

14. Zhu, Z. et al. Causal associations between risk factors and common diseases inferred from GWAS summary data. Nat Commun 9, 224 (2018).

15. Burgess, S. et al. Guidelines for performing Mendelian randomization investigations. Wellcome Open Res 4, 186 (2019).

16. Qi, G. \& Chatterjee, N. A comprehensive evaluation of methods for Mendelian randomization using realistic simulations and an analysis of 38 biomarkers for risk of type 2 diabetes. International Journal of Epidemiology (2021). 
17. DeFronzo, R.A. et al. Type 2 diabetes mellitus. Nat Rev Dis Primers 1, 15019 (2015).

18. Bhupathiraju, S.N. \& Hu, F.B. Epidemiology of Obesity and Diabetes and Their Cardiovascular Complications. Circ Res 118, 1723-35 (2016).

19. Bray, G.A. et al. The Science of Obesity Management: An Endocrine Society Scientific Statement. Endocr Rev 39, 79-132 (2018).

20. Chiu, C.J., Li, S.L., Wu, C.H. \& Du, Y.F. BMI Trajectories as a Harbinger of PreDiabetes or Underdiagnosed Diabetes: an 18-Year Retrospective Cohort Study in Taiwan. J Gen Intern Med 31, 1156-63 (2016).

21. Khera, A.V. \& Kathiresan, S. Genetics of coronary artery disease: discovery, biology and clinical translation. Nat Rev Genet 18, 331-344 (2017).

22. Reiner, Z. Hypertriglyceridaemia and risk of coronary artery disease. Nat Rev Cardiol 14, 401-411 (2017).

23. Malakar, A.K. et al. A review on coronary artery disease, its risk factors, and therapeutics. J Cell Physiol 234, 16812-16823 (2019).

24. Flint, A.C. et al. Effect of Systolic and Diastolic Blood Pressure on Cardiovascular Outcomes. N Engl J Med 381, 243-251 (2019).

25. Toulopoulou, T. et al. Reciprocal causation models of cognitive vs volumetric cerebral intermediate phenotypes for schizophrenia in a pan-European twin cohort. Mol Psychiatry 20, 1386-96 (2015).

26. Nonrecursive Models: Endogeneity, Reciprocal Relationships, and Feedback Loops. (Thousand Oaks, California, 2011).

27. Yang, J. et al. Conditional and joint multiple-SNP analysis of GWAS summary statistics identifies additional variants influencing complex traits. Nat Genet 44, 369-75, S1-3 (2012).

28. Zhang, Y., Qi, G., Park, J.H. \& Chatterjee, N. Estimation of complex effect-size distributions using summary-level statistics from genome-wide association studies across 32 complex traits. Nat Genet (2018).

29. Buckland, S.T., Burnham, K.P. \& Augustin, N.H. Model Selection: An Integral Part of Inference. Biometrics 53, 603-618 (1997).

30. Varin, C., Reid, N. \& Firth, D. AN OVERVIEW OF COMPOSITE LIKELIHOOD METHODS. Statistica Sinica 21, 5-42 (2011).

31. Yang, J., Lee, S.H., Goddard, M.E. \& Visscher, P.M. GCTA: a tool for genomewide complex trait analysis. Am J Hum Genet 88, 76-82 (2011).

32. Chang, C.C. et al. Second-generation PLINK: rising to the challenge of larger and richer datasets. Gigascience 4, 7 (2015).

33. Wu, T. \& Sham, P.C. On the Transformation of Genetic Effect Size from Logit to Liability Scale. Behav Genet 51, 215-222 (2021).

34. Gillett, A.C., Vassos, E. \& Lewis, C.M. Transforming Summary Statistics from Logistic Regression to the Liability Scale: Application to Genetic and Environmental Risk Scores. Human Heredity 83, 210-224 (2018).

35. Nelson, C.P. et al. Association analyses based on false discovery rate implicate new loci for coronary artery disease. Nat Genet 49, 1385-1391 (2017).

36. Malik, R. et al. Multiancestry genome-wide association study of 520,000 subjects identifies 32 loci associated with stroke and stroke subtypes. Nat Genet 50, 524-537 (2018). 
37. Mahajan, A. et al. Fine-mapping type 2 diabetes loci to single-variant resolution using high-density imputation and islet-specific epigenome maps. Nat Genet 50, 1505-1513 (2018).

38. Warrington, N.M. et al. Maternal and fetal genetic effects on birth weight and their relevance to cardio-metabolic risk factors. Nat Genet 51, 804-814 (2019).

39. Yengo, L. et al. Meta-analysis of genome-wide association studies for height and body mass index in approximately 700000 individuals of European ancestry. Hum Mol Genet 27, 3641-3649 (2018).

40. Lu, Y. et al. New loci for body fat percentage reveal link between adiposity and cardiometabolic disease risk. Nat Commun 7, 10495 (2016).

41. Lagou, V. et al. Sex-dimorphic genetic effects and novel loci for fasting glucose and insulin variability. Nat Commun 12, 24 (2021).

42. Willer, C.J. et al. Discovery and refinement of loci associated with lipid levels. Nat Genet 45, 1274-1283 (2013).

43. Han, X. et al. Using Mendelian randomization to evaluate the causal relationship between serum C-reactive protein levels and age-related macular degeneration. Eur J Epidemiol 35, 139-146 (2020).

44. Liu, M. et al. Association studies of up to 1.2 million individuals yield new insights into the genetic etiology of tobacco and alcohol use. Nat Genet 51, 237-244 (2019).

45. Evangelou, E. et al. Genetic analysis of over 1 million people identifies 535 new loci associated with blood pressure traits. Nat Genet 50, 1412-1425 (2018).

46. Howard, D.M. et al. Genome-wide meta-analysis of depression identifies 102 independent variants and highlights the importance of the prefrontal brain regions. Nat Neurosci 22, 343-352 (2019). 
Figures

Figure 1

a

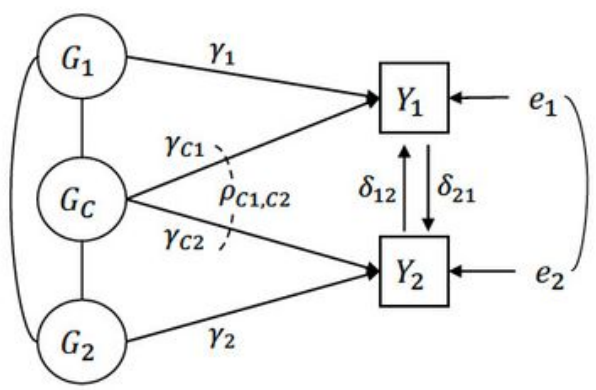

b

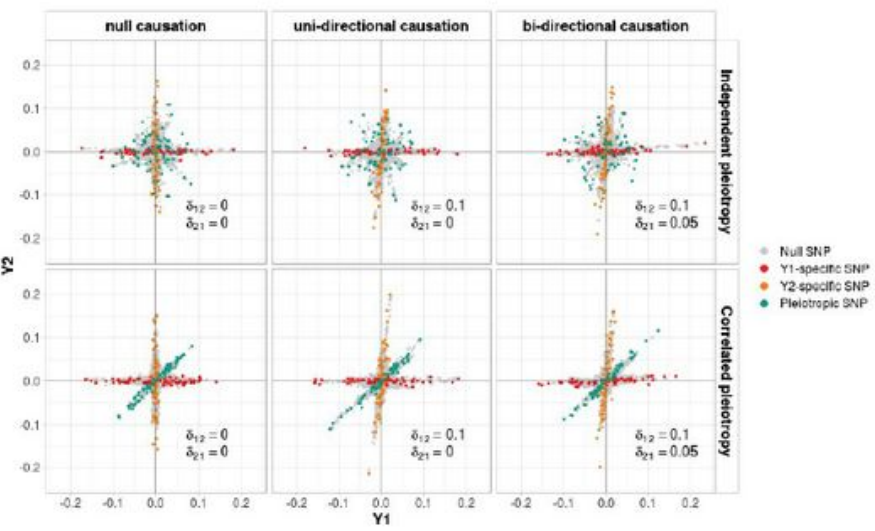

Figure 1. Schematic diagram of $\mathrm{MRCl}$. a, $Y_{1}$ and $Y_{2}$ represent a pair of phenotypes. Genotypes can be divided into four components: $Y_{1}$-specific causal $\operatorname{SNPs}\left(G_{1}\right), Y_{2}$ specific causal SNPs $\left(G_{2}\right)$, pleiotropic causal SNPs $\left(G_{C}\right)$ and null SNPs $\left(G_{0}\right.$, not shown). Lines connecting these genotypes represent the LD correlation between SNPs. Arrow lines from genotype to phenotype represent the direct effect of corresponding SNPS $\left(\gamma_{1}, \gamma_{2}, \gamma_{C 1}\right.$ and $\left.\gamma_{C 2}\right)$ on the phenotypes. The covariance $\left(\rho_{C 1, C 2}\right)$ between $\gamma_{C 1}$ and $\gamma_{C 2}$ is allowed. Arrow lines between the two phenotypes represent the reciprocal causal paths $\left(\delta_{12}\right.$ and $\left.\delta_{21}\right)$. Non-additive genetic effects on phenotypes are represented by $e_{1}$ and $e_{2}$. In real situations, one or two components in the model could be absent and our method could handle these sub-model scenarios in a robust way. $\mathbf{b}$, illustration of several representative simulation scenarios. $x$-axis and $y$-axis show the standardized effect size estimates for GWAS $Y_{1}$ and $Y_{2}$ respectively. Green, red, orange and grey points represent pleiotropic, $Y_{1}$-specific, $Y_{2}$-specific and null SNPs in the simulation respectively. For null causation, $\delta_{12}=\delta_{21}=0.0$; for unidirectional causation, $\delta_{12}=0.1, \delta_{21}=0.0$; for bi-directional causation, $\delta_{12}=0.1$, $\delta_{21}=0.05$. For independent pleiotropy, $\rho_{C 1, c 2}=0.0$; for correlated pleiotropic effects, $\rho_{C 1, C 2}=0.1$. In these plots, the mixing proportions for non-null components were set as $\pi_{1}=\pi_{2}=\pi_{c}=1 \times 10^{-4}$, and the heritability contributed by $Y_{1}$ specific, $Y_{2}$-specific and pleiotropic SNPs were set as $0.3,0.3$ and 0.1 respectively. (see Supplementary Figure S1 for other simulated scenarios)

\section{Figure 1}

Please see the figure for the caption. 
Figure 2

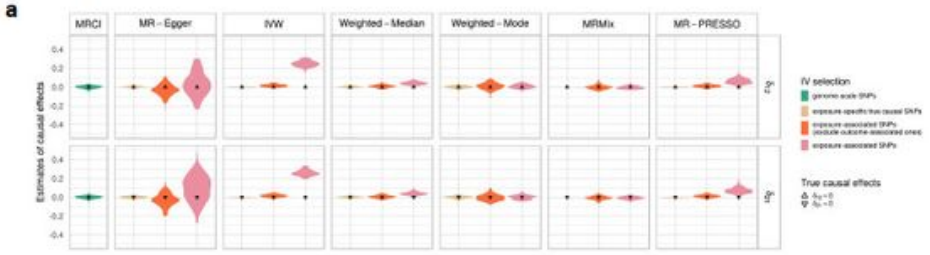

b

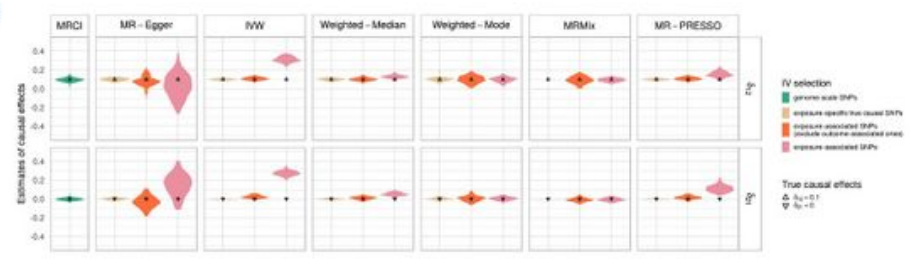

c

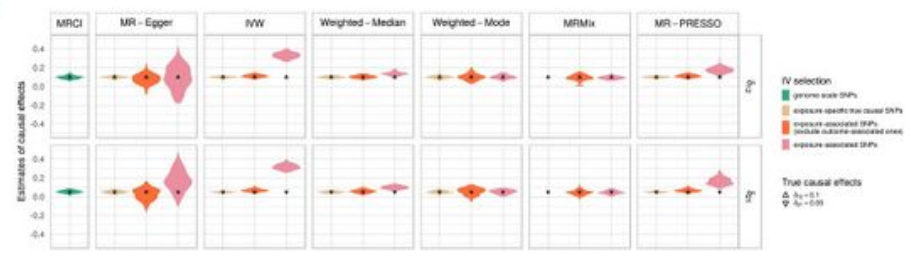

d

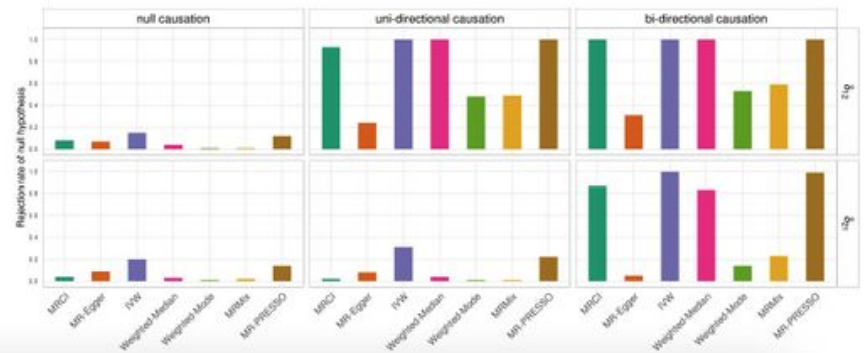

Figure 2. Comparison of estimates for the reciprocal causal effect by our method and instrumental variable (IV)-based MR methods in simulations with correlated pleiotropy. a, estimates in null causation scenarios $\left(\delta_{12}=\delta_{21}=0.0\right)$. b, estimates in uni-directional causation scenarios $\left(\delta_{12}=0.1\right.$ and $\left.\delta_{21}=0.0\right)$. c, estimates in bidirectional causation scenarios $\left(\delta_{12}=0.1\right.$ and $\left.\delta_{21}=0.05\right)$. d, rejection rates of null hypothesis in bi-directional, uni-directional and null scenarios. In plots a, b and c, our method took genome-scale SNPs for estimation and produced nearly unbiased estimates in different scenarios. For MR methods, IVs were selected in three ways: (1) use the exposure-specific true causal SNPs in the simulation as IVs; (2) use exposure-associated SNPs ( $\mathrm{p}$-value $<5 \times 10^{-8}$ ) after clumping but exclude potential outcome-associated SNPs (defined as p-value $<5 \times 10^{-5}$ with outcome); (3) use significant exposure-associated SNPs after clumping regardless of their association with outcome. In plot $\mathbf{d}$, the exclusion criteria were applied to IV-based MR methods and our method shows well-controlled Type I error rates and adequate power. In these plots, the true values of $\delta_{12}$ and $\delta_{21}$ are indicated by up- and down-pointing triangles, respectively; $\rho_{C 1, C 2}$ for the correlated pleiotropy is 0.1 ; the mixing proportions for non-null components are $\pi_{1}=\pi_{2}=\pi_{c}=1 \times 10^{-4}$; the heritability contributed by $Y_{1}$-specific, $Y_{2}$-specific and pleiotropic SNPs are $0.3,0.3$ and 0.1 respectively. (Note: The selection of exposure-specific true causal SNPS was not applied to MRMix due to its assumption of a normal-mixture distribution)

\section{Figure 2}

Please see the figure for the caption. 


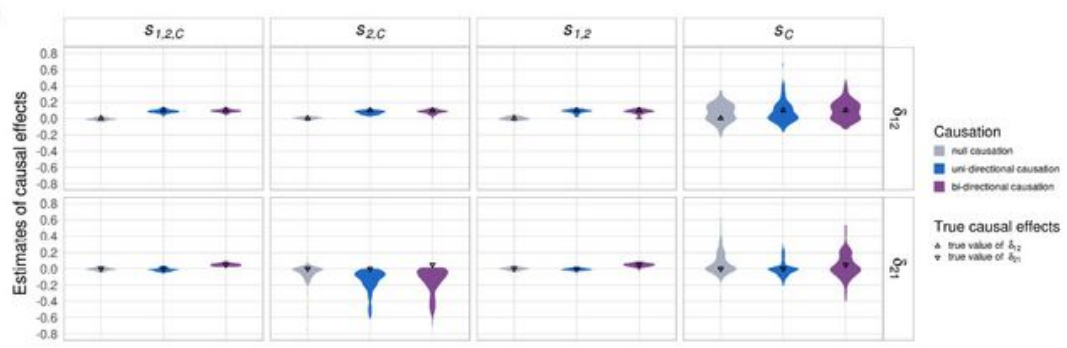

b

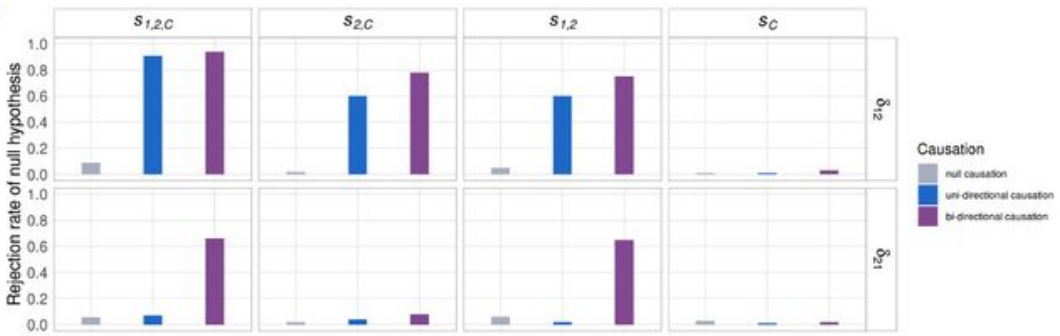

Figure 3. Estimation after model averaging. a, final estimates of causal effects under bi-directional, uni-directional and null causations in four scenarios $\left(s_{1,2, C}, s_{2, c}, s_{1,2}\right.$ and $s_{C}$ ). When the exposure-specific SNPs exist, the final estimates after model averaging still produced nearly unbiased estimates in simulated scenarios. For null

causation (grey), $\delta_{12}=\delta_{21}=0.0$; for uni-directional causation (blue), $\delta_{12}=0.1$ and $\delta_{21}=0.0$; for bi-directional causation (purple), $\delta_{12}=0.1$ and $\delta_{21}=0.05$. The true causation values of $\delta_{12}$ and $\delta_{21}$ are indicated by up- and down-pointing triangles, respectively. b, rejection rate of null hypothesis of the final $\delta_{12}$ and $\delta_{21}$ estimates after model averaging in different scenarios. For zero-effect causal direction, the Type I error rates are well-controlled; for non-zero-effect causal direction, the presence of the exposure-specific SNPs shows reasonably good power, and the absence of the exposure-specific SNPs shows conservative power of estimation. In the simulations, the mixing proportion of the present component was $1 \times 10^{-3}$; the pleiotropic effects were correlated $\left(\rho_{c 1, c 2}=0.1\right)$; the heritability contributed by $Y_{1}$ specific, $Y_{2}$-specific and pleiotropic SNPs (if present in the sub-model scenario) were $0.3,0.3$ and 0.1 , respectively.

\section{Figure 3}

Please see the figure for the caption. 
Figure 4

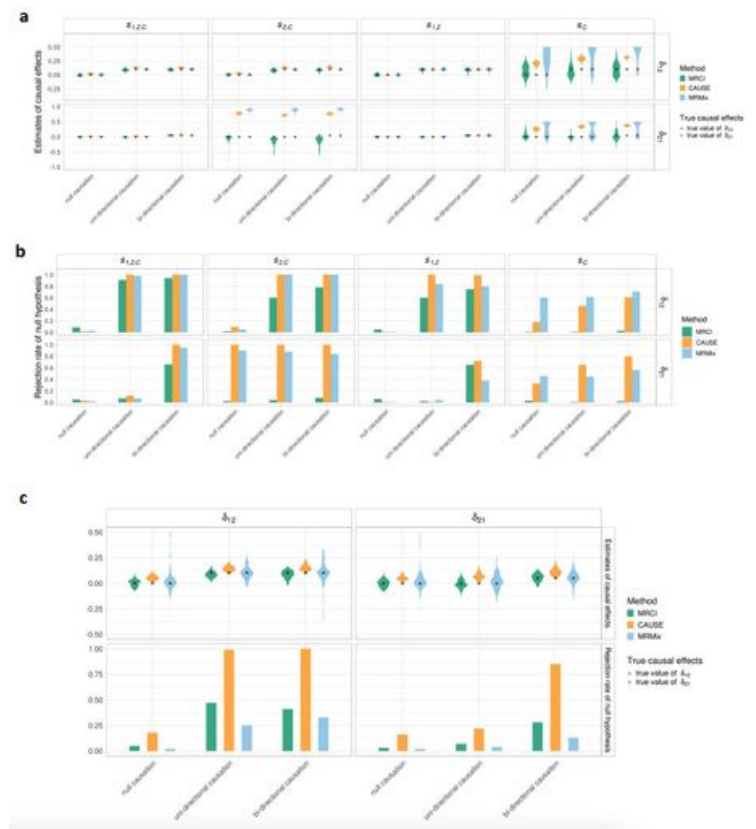

Figure 4. Estimation comparison with CAUSE and MRMix. a, estimates from our method (green), CAUSE (yellow) and MRMix (blue) under bi-directional, unidirectional and null causations in four scenarios $\left(s_{1,2, C}, s_{2, c}, s_{1,2}\right.$ and $\left.s_{C}\right)$. CAUSE and MRMix produced severely over-estimated causal effects when exposure-specific SNPs are absent in the sub-model $s_{2, C}$ and $s_{C}$. For null causation, $\delta_{12}=\delta_{21}=0.0$; for uni-directional causation, $\delta_{12}=0.1$ and $\delta_{21}=0.0$; for bi-directional causation, $\delta_{12}=0.1$ and $\delta_{21}=0.05$. The true causation values of $\delta_{12}$ and $\delta_{21}$ are indicated by up- and down-pointing triangles, respectively. $\mathbf{b}$, rejection rate of null hypothesis for $\delta_{12}$ and $\delta_{21}$ estimates from our method (green), CAUSE (orange) and MRMix (blue) in different simulated scenarios. CAUSE and MRMix produced inflated Type I error rates for the causal direction where exposure-specific SNPs were absent $\left(s_{2, c}\right.$ and $s_{C}$ ). c, estimates of causal effects and rejection rate of null hypothesis from our method (green), CAUSE (orange) and MRMix (blue) under bi-directional, unidirectional and null causations in $s_{1,2, C}$ scenario with small sample sizes $(20,000$ individuals). Decreased GWAS power led to over-estimates for CAUSE and larger estimation variance for MRMix. Our method produced nearly unbiased correct Type I error rates. In these results, the estimates of our method came from the final estimates after model averaging; P-value thresholds for CAUSE and MRMix were $1 \times 10^{-3}$ and $5 \times 10^{-8}$, respectively. In the simulations, the mixing proportion of the present component was $1 \times 10^{-3}$; the pleiotropic effects were correlated $\left(\rho_{C 1, C 2}=0.1\right)$; the heritability contributed by $Y_{1}$-specific, $Y_{2}$-specific and pleiotropic SNPs (if present in the sub-model scenario) were $0.3,0.3$ and 0.1 , respectively.

\section{Figure 4}

Please see the figure for the caption. Figure 5

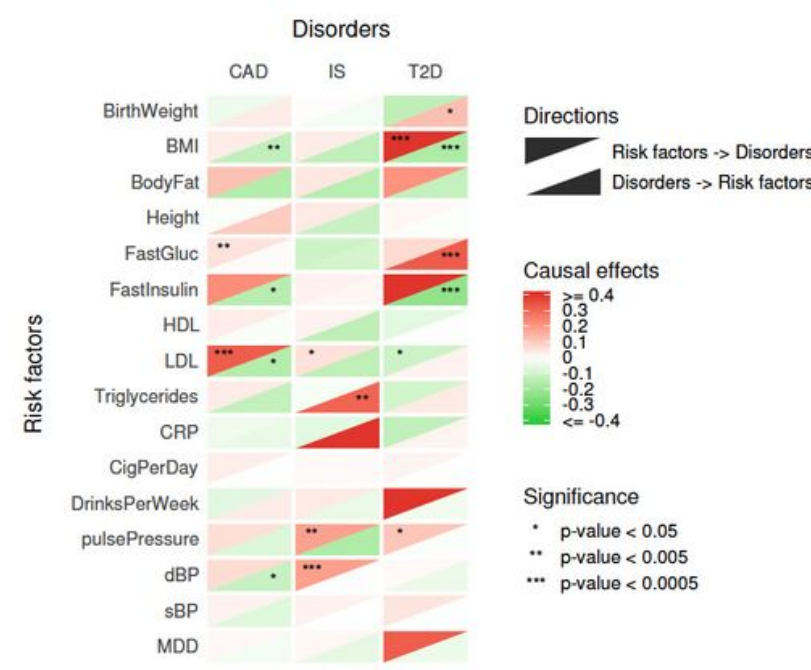

Figure 5. Summary of estimates for three common diseases and sixteen risk factors. In the figure, the upper triangle in a box represents the causal direction from the disorder to the risk factor, and the lower triangle represents the reverse causal direction. Darker color represents stronger causation estimates. Stars in the triangle represent different significance levels of the estimation. Abbreviations: CAD coronary artery disease; IS - any ischemic stroke; T2D - type 2 diabetes; BirthWeight - birth weight; BMI - body mass index; BodyFat - body fat percentage; FastGluc fasting glucose level; FastInsulin - fasting insulin level; HDL - high-density lipoprotein cholesterol level; LDL - low-density lipoprotein cholesterol level; Triglycerides triglyceride level; CRP - C-reactive protein level; CigPerDay - cigarettes per day;
DrinksPerWeek - drinks per week; pulsePressure - pulse pressure; dBP - diastolic blood pressure; sBP - systolic blood pressure; MDD - major depressive disorder.

\section{Figure 5}

Please see the figure for the caption.

\section{Supplementary Files}


- SuppNote.pdf

- SuppFigures.pdf

- SuppTables.pdf 